\title{
La salud como un derecho fundamental, implementada en un nuevo modelo de salud en Colombia.
}

\section{Health as a fundamental right, implemented in a new model of health in Colombia}

\author{
Autores: Iliana Marcela Fortich Lozano ${ }^{1}$ \\ Correspondencia: ilianamarcelafortich@hotmail.com
}

\begin{abstract}
RESUMEN
Este artículo de revisión tiene el propósito de explicar cómo se consiguió elevar el derecho a la salud con un rango de derecho fundamental en Colombia. A la luz de la Constitución Política colombiana podemos afirmar que contiene una normatividad amplia sobre la Seguridad Social, pero no precisando la salud como derecho fundamental. Por ello, es perentorio explicar de dónde proviene el alcance de derecho fundamental al derecho a la salud, y prueba de ello lo tienen el artículo 44 de nuestra carta magna donde nos muestra cómo se consagra como derecho fundamental de los niños el derecho a la salud y la Seguridad Social.
\end{abstract}

\section{Palabras Claves}

Salud, Derecho Fundamental, Seguridad Social, Constitución.

\begin{abstract}
This revision paper aims to explain how they managed to raise the right to health with a range of fundamental rights in Colombia. In light of the Colombian Constitution we can affirm that contains extensive regulations on social security, but not requiring health as a fundamental right. It is therefore imperative to explain from where the scope of the fundamental right to right to health, and the proof we have Article 44 of our Constitution which shows how enshrined as a fundamental right of children the right to health and Social Security.
\end{abstract}

\section{Keywords}

Health Fundamental Law, Social Security, Constitution.

\footnotetext{
${ }^{1}$ Profesora de la Corporación Universitaria Rafael Nuñez y la Universidad Libre, Sede Cartagena. Abogada de la CUC. C.Mg. Derecho, Universidad de Cartagena. Especialista en Seguridad Social de la Universidad de Cartagena, coinvestigadora y miembro del grupo de investigación teoría jurídica y derechos fundamentales "phrónesis". Email: ilianamarcelafortich@hotmail.com.
} 


\section{Introducción.}

El artículo 46 de la constitución política colombiana garantiza a las personas de la tercera edad los servicios de Seguridad Social Integral. Precisamente el articulo 47 nos muestra una política de previsión, rehabilitación e integración social para los discapacitados físicos y psíquicos, y el articulo 48 siendo el más importante de nuestra Constitución, hace referencia al derecho a la Seguridad Social Integral, como un derecho irrenunciable, que es un servicio público de carácter obligatorio que se prestara bajo la dirección, coordinación del Estado.

El artículo 49 garantiza el acceso a los servicios de promoción, protección y recuperación de la salud a todas las personas, el artículo 50 señala que todos los niños menores de un año que no estén cubiertos por algún tipo de protección, tendrán derecho a recibir servicios de salud gratuita en todas las instituciones de salud subsidiadas por el Estado. Los artículos 356 y 357 modificados por los Actos Legislativos 01 de 2001 y 04 de 2007, crean el Sistema General de Participaciones de los Departamentos, Distritos y Municipios, que los recursos asignados a estos, deberán ser distribuidos teniendo como prioridad la destinación a los servicios de salud y la educación. Como podemos observar la carta no menciona en si el derecho a la salud como derecho fundamental para todas las personas; existe una contradicción entre el articulo 44 y el 48 de la Constitución donde el primero nos menciona que la salud es un derecho fundamental pero haciendo claridad que solo para los niños, y el artículo 48 señala que la Seguridad Social es un servicio público de carácter obligatorio, es decir que la salud, no sería un derecho fundamental para los adolescentes y los adultos.

En 1992 la Corte Constitucional se pronuncia al respecto de la salud como derecho fundamental a través de la Sentencia T-561 de 1992 donde considera la 
Seguridad Social un derecho fundamental, cuando su no reconocimiento quebranta derechos fundamentales como la vida, la dignidad humana, la integridad física y moral, el libre desarrollo de la personalidad y derechos de la persona de la tercera edad. De igual forma, la Corte Constitucional se ha pronunciado en muchas sentencias sobre la Seguridad Social Integral reiterando que es un derecho fundamental, ya sea en relación a la salud o a las pensiones en general. Además la Constitución Colombiana, presenta contradicciones en relación a la Seguridad Social, con lo que establece la normatividad internacional, ya que el artículo 22 de la Declaración Universal de los Derechos Humanos y los artículos 9 y 12 del Pacto Internacional de Derechos Económicos, Sociales y Culturales le reconocen una prioridad a la Seguridad Social, mientras que en nuestra Constitución la Seguridad Social está contemplada entre los derechos sociales, económicos y culturales, como derechos de segunda generación.

A lo largo del desarrollo de este trabajo, podremos obtener conocimiento sobre el recorrido que ha tenido la salud, en nuestro país para llegar a estar completamente regulado por una norma relativamente nueva donde a través de una ley, el presidente de la republica declara la salud como derecho fundamental para todos los colombianos.

\section{Método.}

El presente artículo se enmarca en un tipo de investigación descriptiva. Con un enfoque cualitativo- hermenéutico y las herramientas metodológicas utilizadas son las documentales. 


\section{Resultados Y Discusión}

\section{Modelo actual de Salud}

El modelo de seguridad social en salud, implementado por la Ley 100 de 1993, está establecido según los parámetros del informe del Banco Mundial que recomendó un diseño de servicios de salud para los países de ingreso bajo y envía de desarrollo.

Es interesante mencionar un acápite del tratadista Rodríguez Mesa Rafael, en su texto

El autor nos señala cuatro fases que se recomendaron para este modelo de salud:

"La primera consistió en reforzar la noción del fracaso de lo publico en la oferta de servicios (ineficiente), acompañándola de la tesis de que la salud corresponde al ámbito de lo privado, para puntualizar que la mejor alternativa es la oferta privada de la salud.

La segunda consistió en desarrollar un nuevo indicador: años de vida ajustada por discapacidad o años de vida saludable perdidos. Este nuevo indicador se acompañó de la noción de servicios clínicos mínimos que estarían definidos, entre otras cosas, por criterios de costo/beneficio (en unidades monetarias), costo-efectividad (uso racional de recursos) y costo/utilidad (años de vida ajustados a la calidad). (rodriguez, 2015, pág. 363)

La tercera fue impulsar la tesis de que el problema central de la crisis hospitalaria - y por lo tanto de los sistemas de salud- es que las formas de administrar de lo público son per se ineficientes y que la solución es adoptar políticas de administración hospitalaria con indicadores y recursos propios de la empresa privada. 
La cuarta fue establecer políticas financieras tendientes a la autofinanciación de los servicios de salud, en un contexto de reducción progresiva de los recursos del estado para este sector $\mathrm{y}$ de establecimiento de formas de pago directo $\mathrm{y} / \mathrm{o}$ de formas compartidas de financiación por parte del usuario de los servicios adicionales al pago de las cotizaciones de rigor

Con este propósito se creó un intermediario en la oferta de servicios, encargado de recolectar las cotizaciones y de contratar servicios de hotelería, en detrimento de las investigaciones sobre las condiciones de salud de la población."

Como podemos observar en la explicación anterior que hace el autor citado anteriormente, se muestra con claridad que las directrices que planteo el Banco mundial establecen parámetros reales que se adoptaron con la Ley 100 de 1993, donde se organiza el sistema de salud de los colombianos con la creación de nuevas entidades de salud, como Las Empresas Promotoras de salud EPS, LAS Instituciones de Prestadoras de Servicios de Salud IPS, de igual forma se crees EI Plan Obligatorio de Salud POS, y el ofrecimiento por las mismas EPS, y las aseguradoras autorizadas para ofrecer servicios adicionales de salud, conocidos como PACS Planes Adicionales Complementarios de Salud o Medicina Pre pagada, financiadas con el pago de una cotización adicional a la establecida por el estado por el mismo afiliado al sistema de salud.

Con la adopción de este modelo de salud se entregó el sector de la salud, el de las pensiones, los riesgos laborales y los servicios sociales complementarios al sector financiero privado. Por esta razones expuestas es notorio para todos los colombianos que el sistema de salud se ha convertido en una ganancia de utilidades para los intermediarios del sector privado, que inicialmente se planteó que el rendimiento financiero mayor, seria para los usuario, pero el resultado ha sido la inversión de los recursos recolectados a través de las cotizaciones, para generar las grandes ganancias para los intermediarios y las perdidas 
trasladárselas a los afiliados contribuyentes a través del Fondo de Solidaridad Y Garantía.

Para mayor ilustración de los lectores en este tema, a continuación en una breve explicación se relatara como funciona, se financia, y los organismos que lo conforman para su dirección, vigilancia y control; y los órganos que lo administran y lo financian. el sistema de salud actualmente en Colombia.

\section{1 composición básica del sistema de salud}

Está conformado por dos sectores:

- Sector Privado: Conformado por Las Empresa Promotora de Salud EPS Y Las Instituciones Prestadoras de Servicios de Salud IPS.

- Las EPS organiza garantiza el Plan Obligatorio de Salud POS a los afiliados, son las que administran el Régimen Contributivo, se encargan de la afiliación, del recobro de las cotizaciones, y de la Unidad per cápita UPC; y contratan servicios de salud con las IPS y profesionales independientes especializados, para la prestación de los servicios de salud

- Las IPS son los hospitales, clínicas, laboratorios y consultorios.

- Sector público: Está conformado por las Direcciones Seccionales Distritales y Locales de Salud, por las IPS, EPS públicas y las Empresas Sociales del Estado ESE.

- Las primeras se encargan de la sostenibilidad financiera de las IPS públicas, garantizar el acceso, calidad y servicios. Optimizar el uso de los recursos, promover enfoques de atención al usuario.

- Las ESE se encargan de prestar servicios respectivo nivel de atención en ambos regímenes, responsabilidad atención de la salud población vulnerable (vinculados), contratación de servicios ambos regímenes.

\subsection{Riesgos que atiende el sistema}

- Enfermedad general 
- Afecciones de la salud que no guarden relación con el trabajo

- Maternidad

\subsection{Responsabilidad en la salud publica}

Son responsables en la salud pública:

a) En el primer nivel: Municipio, Distritos, Hospitales Locales y Centros y puestos de Salud.

b) En el segundo y tercer nivel: Departamentos Distritos Especiales, Hospitales Regionales, Universitarios y Especializados.

\subsection{Regímenes del Sistema de Seguridad Social en Salud}

Está conformado por:

- Régimen Contributivo: Población con capacidad de pago, personas que tengan un contrato laboral, o trabajadores independientes.

- Régimen Subsidiado: Población vulnerable, subsidiada por el estado total o parcialmente.

- Régimen de los Vinculados o Participantes: Población más vulnerable que no tienen la calidad de beneficiarios del régimen subsidiado, y temporalmente reciben servicios de salud.

\subsection{Integrantes en el Sistema General de Seguridad Social en Salud}

a) Órganos de Dirección, Vigilancia y Control:

- Ministerio de Salud y del Trabajo y la Protección social

- Consejo Nacional de Seguridad Social en Salud

- La Superintendencia Nacional en Salud

-Comisión de Regulación en Salud CRES

b) Órganos de Administración y Financiación

- Las Empresas Promotoras de Salud EPS

- Las Direcciones, Distritales y Locales de Salud

- Las Instituciones Prestadoras de Servicios de Salud IPS

- El Fondo de Solidaridad y Garantía FOSYGA

- Los Empleadores, los Trabajadores y sus Organizaciones 
- Los Empleadores del Sistema General de Seguridad Social en Salud

- Los Comités de Participación Comunitarias COPAGOS

En relación a la inspección y vigilancia y control del sistema de salud, es notorio que padece de muchas falencias ya que esta funciones son manejadas de una forma descoordinada, y desorganizadamente, debido a la falta de claridad entre los distintos funcionarios que les compete esta tarea, además la atención está más enfocada a la parte financiera y no a los problemas reales de salud que se les presentan a los usuarios.

El tratadista Rodríguez Mesa Rafael ("Estudios Sobre Seguridad Social”, Editorial Universidad del Norte, Barranquilla, 2015, pag 366-367), en su texto nos muestra una recopilación de informes y consolidados proyectados por la Procuraduría y la Defensoría del Pueblo, relacionados con el tema de la salud en Colombia (El tiempo, Edición del 13 de Agosto de 2006, pág. 1-6), donde nos indica la siguiente información sobre el tema a tratado.

"En mayo de 2008 el Procurador General de la Nación presento el informe ("El derecho a la salud en perspectiva de derechos humanos y el sistema de inspección y vigilancia y control del Estado Colombiano en Materia de queja de salud.")

En este informa la Procuraduría señala la persistencia de profundas injusticias en el sistema, el no logro de la cobertura universal y el estancamiento o retroceso en prevención de enfermedades y vacunación. Después de años han vuelto a aparecer casos de rabia o de fiebre amarilla y varios índices de vacunación continúan por debajo de las metas.

El informe presenta que el $73 \%$ de las tutelas se presentan por usuarios del Régimen Contributivo, mientras que solo 2,9\% son del subsidiado.

La Defensoría del Pueblo señalo en el 2006 que el grueso de las acciones de tutela se interpone para reclamar medicamentos o procedimientos que están incluidos en el POS. 
La Defensoría del Pueblo público en agosto de 2014 un informe sobre la acción de tutela en relación con el derecho a la salud y que en 2013 se interpusieron 115.014 acciones de tutela (834 más que en 2012) para reclamar medicamentos y citas médicas. De acuerdo con el estudio, 890 de 1.123 municipios del país se interpuso por lo menos una tutela que reclamaba el derecho a la salud y en 475 se constituyó en la solicitud más frecuente. Lo más grave de la situación es que el $69,96 \%$ de las acciones de tutela se interpuso para pedir servicios, medicamentos y otros contenidos en el POS. Según el Informe de la Defensoría del Pueblo, los pobres son los más afectados por la negación de los servicios del POS. La prueba es que en el Régimen Subsidiado el $75,63 \%$ de las tutelas fue para pedir servicios incluidos en el POS. En 2013 en el régimen subsidiado se presentaron por juez constitucional el $28,72 \%$ de los tratamientos integrales, el $17,46 \%$ para citas con especialistas y el $16,42 \%$ respecto de medicamentos. El $85,46 \%$ de los tratamientos pedidos a través de la tutela estaban incluidos en el POS. En el régimen contributivo el panorama el similar: $64,86 \%$ de las tutelas reclamaban servicios incluidos en el POS. De este porcentaje, el $44 \%$ de las demandas se instauro para pedir tratamientos integrales POS y el 15,02\% para conseguir una cita médica."

En este informe remitido por la Procuraduría y La defensoría del Pueblo, podemos observar la gravedad del estado de nuestro sistema de salud actualmente, la salud se ha convertido en un negocio, para los intermediarios, y los servicios incluidos en el Plan Obligatorio de Salud POS, al parecer en la práctica, es como si los usuarios no tuviesen ningún derecho al acceso de los servicios de salud. Las Empresas Promotoras de Salud, deberían ser suprimidas ya que no están cumpliendo con su papel, simplemente se les debe relevar de intermediarias financieras del sistema de salud. Luego de la expedición de la Ley 1438 de 20011, las acciones de tutela en un alto porcentaje son negadas a pesar de que los afiliados tenían derecho a ellas por estar incluidos los tratamientos, procedimientos y medicamentos en el POS. 
A continuación brindaré una breve definición de lo que es el Plan Obligatorio de Salud POS, y el Fondo de Solidaridad Y garantía FOSYGA. Participantes de gran importancia en nuestro sistema de salud.

PLAN OBLIGATORIO DE SALUD POS: Plan integral de salud, atención preventiva, medico quirúrgica y medicamentos esenciales.

Fondo de Solidaridad y Garantía FOSYGA:

Es una cuenta del Estado adscrita al Ministerio d Salud y la Protección Social, manejada por encargo fiduciaria, sin personería jurídica y maneja los recursos destinados a la inversión de salud.

2. reforma a la salud ley 1438 de 2011

La Ley 1438 de 2011, introdujo una nueva reforma para nuestro sistema de salud, ley que entro en vigencia en el mes de enero de 2012, dicha ley fue creada con el propósito de mejorar nuestro sistema de salud, esta nos trae la inclusión de nuevos principios rectores en el sistema de salud, con el fin de dar cumplimiento a los objetivos del sistema de salud como crear condiciones de acceso a los servicios en todo los niveles de atención, introducir la participación de diferentes y nuevas entidades, y propender por la cobertura familiar.

La ley 1438 de 2011, nos menciona los siguientes principios:

- Universalidad: Cobertura en todas las etapas de la vida

- Solidaridad: Mutuo apoyo

- Igualdad: Sin discriminación

- Obligatoriedad: Todos los residentes en Colombia

- Prevalencia de Derechos: Niños, adolescentes, mujeres en estado de embarazo

- Enfoque Diferencial: Características particulares, edad, raza, violencia

- Equidad: Acceso plan de beneficios sin tener en cuenta capacidad de pago

- Calidad: Atención humanizada 
- Eficiencia: Calidad de vida

- Participación Social: Intervención de la comunidad

- Progresividad: Gradualidad plan de beneficios

- Libre Escogencia: Afiliados

- Sostenibilidad: Recursos destinados por la ley

- Transparencia: Publicas, claras y visibles

- Descentralización Administrativa: Organización descentralizada

- Complementariedad y Concurrencia: Distintos niveles territoriales se complementan

- Corresponsabilidad: Propender por su auto cuidado

- Irrenunciabilidad: El derecho a la seguridad social es irrenunciable.

- Intersectorialidad: Acción conjunta coordinada de los sectores

- Prevención: Precaución

- Continuidad: Vocación de permanencia

Además de la inclusión de estos nuevos principios en el sistema de salud con la nueva reforma que se dio en el 2011, también se crearon varias entidades que en la Ley 100 de 1993, no se establecieron en su organización. Estas nuevas entidades de salud se crean con el objetivo del mejoramiento del sistema, pero muy a pesar de su introducción en nuestro sistema de salud, no se ha manifestado en la práctica, el cumplimiento para la cual fueron creados, ya que los intermediarios del sistema hacen caso omiso a los principios establecidos en la reforma, y de igual forma no funcionan las entidades nuevas como nos señala la ley. Mencionaremos algunas de estas nuevas entidades creadas por la ley 1438 de 2011.

\subsection{Plan decenal para la salud publica}

Creado por la ley 1438 de 2011, por el Ministerio de la Protección Social, hoy Ministerio de Salud y la Protección Social, con el propósito de establecer un proceso amplio de participación social en el sistema de salud, crear nuevas estrategias de atención primaria en salud, y al mismo tiempo, confluir las políticas 
sectoriales para el mejoramiento de la salud, incluyendo la salud mental, que esta debe contar con una garantía del ejercicio pleno del derecho a la salud de todos los colombianos, mediante una atención integral, la cual debe hacer parte del Plan de Beneficios y la implementación, seguimiento y evaluación de la política nacional de salud mental.

\subsection{Observatorio Nacional de Salud}

Este nuevo organismo del sistema de salud, fue creado por la Ley 1438 de 2011, con la reforma a la salud, el observatorio es el responsable del monitoreo de la salud pública para cada municipio y departamento, además se encarga del seguimiento de la salud de los colombianos; y sirve de soporte técnico al país en temas de salud. Otras de sus funciones son las de realizar evaluaciones periódicas sobre el estado de salud de las regiones o grupos especiales; y también se encarga de publicar los resultado de las evaluaciones. De igual forma debe fortalecer el sistema de información epidemiológica, con énfasis en las zonas de frontera.

\subsection{Fondo de Salvamento y Garantías para el Sector Salud}

Este fondo también fue creado por la Ley 1438 de 2011, como una cuenta del estado, sin personería jurídica, adscrita al Ministerio de la Protección Social, hoy Ministerio de Salud y la Protección Social, con el fin de asegurar el pago de obligaciones que no puedan asumir las Empresas Sociales del Estado ESE, intervenidas por la Superintendencia Nacional de Salud, se financiara hasta el $20 \%$ del gasto operacional, en el caso de las Empresas Sociales del Estado liquidadas, se pagara hasta el monto que determine el Ministerio de salud y la Protección Social.

2.4. Redes Integradas De Servicios de Salud: Las redes también tienen su aparición en el sistema de salud con la expedición de la Ley 1438 de 2011, fueron creadas con el objetivo de prestar servicios de salud individuales y colectivos, a 
los municipios, distritos, departamentos y la nación. Sus funciones deben ser manejadas en coordinación con las Empresas Promotoras de Salud EPS. La prestación de los servicios de salud se hará a través de las redes integradas de servicios de salud ubicadas en determinadas poblaciones. Las redes contaran con la suficiencia técnica administrativa, financiera requerida para promover, la salud y prevenir enfermedades, y prestar los servicios para la recuperación de la salud a través de los tratamientos y rehabilitación requerida, que demande el cumplimiento eficaz de los planes de beneficio. Las redes se seguirán de acuerdo a la reglamentación que expida del Ministerio de Salud y la Protección Social, quien podrá delegar en los departamentos y distritos.

\section{3. la salud como derecho fundamental}

La salud como derecho fundamental, ha sido el objetivo principal de nuestro sistema de salud, a lo largo de todos estos años luego de la organización de nuestro sistema de seguridad social con la expedición de la Ley 100 de 1993, la esperanza de todos los colombianos es poder tener acceso a la salud sin necesidad de sentirnos como si estuviéramos mendingando una atención médica, la cual debe ser prestada a través de nuestro sistema de salud de una manera humana, eficaz, y con calidad óptima. Nuestra constitución de 1991, no incluyo en su articulado, la salud como un derecho fundamental, simplemente está establecido entre los derechos sociales y culturales, es decir quedan relegado el derecho a la salud como un derecho de segunda generación; lo cual nos ha venido afectando a toda la población colombiana, ya que es habitual que para acceder a un servicio médico, sea tratamiento, procedimiento o medicamentos incluidos en el Plan Obligatorio de Salud POS, a través de la interposición de la acción de tutela invocando la conexidad con el derecho a la vida, para que sea admitida esta demanda, este medio se ha convertido en el pan de cada día de los colombianos, para poder medianamente a través de un fallo judicial, obtener el acceso a un servicio de tratamiento, procedimiento o medicamento, que muchas 
ocasiones cuando llega la sentencia favorable, lastimosamente ya es muy tarde la muerte no dio espera, llego antes del fallo. Es de gran tristeza tener que plasmar una realidad tan cierta y cruel que vivimos los colombianos, muy a pesar que en el año 2015, con la expedición de la Ley Estatutaria 1751 de 2015 se elevó la salud a derecho fundamental, todavía en la actualidad se sigue negando la atención inmediata a algunos procedimientos que no tiene espera y ponen en riesgo la vida de la población colombiana.

Antes de entrar de lleno en el tema citare algunos acápites de varios tratadistas que nos orientan sobre la realidad del tema de la salud.

La declaración de los derechos humanos establece en sus artículos 3 "Toda persona tiene derecho a la vida" y 25.1: "Toda persona tiene derecho a un nivel de vida adecuado que le asegure, así como a su familia, la salud y el bienestar." Los tratadistas Herrera Fragoso Agustín Antonio, Moreno hidalgo Omar, Sotelo Monroy Gabriel en su texto, ("El Derecho a la Salud desde el Derecho Internacional de los Derechos Humanos para México", Editorial, Instituto de Ciencias Jurídicas de Puebla A. C., pag 82-83).

"En este sentido la vida y la salud se articulan como valores morales, como bienes fundamentales (privados y públicos), que deben ser respetados y protegidos. La salud es un derecho, pero un derecho que tiene un precio no solo económico, sino a un triple nivel: social, familiar y personal. La salud es reconocida como un derecho inalienable, sujeto a determinadas condiciones o criterios sociales, políticos, económicos y éticos, que ha pasado meramente privada, que cada uno debía gestionar según sus posibilidades, a convertirse en un problema de dimensión pública y política. La importancia de percibir la salud como un bien privado que cada uno está obligado a respetar y cuidar, también resulta claro que la salud es un bien público, por lo que podría ser catalogada como un derecho básico. 
Como se desarrolló con antelación, la salud es una realidad existencial que debe de aspirar a la mayor plenitud del ser humano, pero también puede ser interpretada como un derecho humano básico, y ahí será necesario establecer las causas necesarias, pues no en vano hay aspectos que socialmente pueden ser imposibles de lograr. Garantizar a todo el mundo el derecho de acceso a una adecuada asistencia médica puede ser correcto, pero el derecho a la salud solo puede asegurarse con el ejercicio de una responsabilidad personal; es decir la salud también es una responsabilidad que cada uno deberá personalmente asumir."

Con relación a lo que nos comentan los autores citados, podemos interpretar que el derecho a la salud es un derecho humano básico, que tenemos todos los individuos; que el estado debe controlar a las entidades que prestan estos servicios de salud, para obtener un logro de fácil acceso y recibir las prestaciones asistenciales por parte de los intermediarios de nuestro sistema de salud, además reiteran los tratadistas, y hacen énfasis en la corresponsabilidad de la salud por cada individuo, cada persona debe adquirir una responsabilidad de cuidar su salud personalmente y familiarmente.

Para Bobbio, Norberto en su texto:

"El fundamento de derechos de los que solo se sabe que son condiciones para la realización de valores últimos es la apelación a dichos valores últimos. Pero los valores últimos, a su vez, no se justifican, se asumen: lo que es último, justamente por su carácter de tal, no tiene fundamento alguno." (bobbio, 1992, pág. 121)

Lo planteado por Bobbio, relacionado con el tema en estudio expresa que el debate del derecho a la salud no debe girar sobre su fundamentación, si no más sobre su promoción, control, y garantía; y la aceptación de la salud como derecho fundamental. 


\subsection{Garantías de los Derechos Sociales}

Como bien es sabido el derecho a la salud en nuestra Constitución Política, no hace parte de los derechos fundamentales, sino que está establecido como un derecho social y aparece como el derecho a la seguridad social en el Capítulo II del Título II de los derechos económicos, sociales y culturales.

Teniendo en cuenta la poca importancia que se le dio a la salud en nuestra constitución, es pertinente agregar este acápite de los siguientes tratadistas Según Morales Leticia en su libro, nos comenta "Todavía me resta considerar una última objeción dirigida contra la estructura de los derechos sociales que, desde una teoría realista, ancla la existencia de los derechos a la previsión de su garantía jurisdiccional, desde esta posición, es habitual sostener que un derecho no garantizado jurisdiccionalmente, aunque este consagrado en una Constitución, es un derecho ficticio o directamente inexistente. Este argumento es ilustrado con frecuencia señalando el caso de los derechos sociales. Se sostiene que en tanto estos derechos carecen, por lo general, de la consagración de una norma jurídica que de modo explícito confiera acciones procesales necesarias para exigir jurisdiccionalmente la conducta debida, se sigue que no pueden ser considerados auténticos derechos." (morales, 2015, pág. 104)

En relación a la autora citada vemos su concepción de un derecho social, en realidad carecen de una norma jurídica explicita y para lograr su cumplimiento se deben utilizar acciones judiciales para poder obtener el resultado buscado; cómo podemos darnos cuenta el derecho a la salud en nuestra constitución está plasmado como derecho social por lo tanto, debemos interponer la acción de tutela que es el mecanismo más rápido, para poder obtener ese resultado, que supuestamente está garantizado constitucionalmente.

Veamos lo que nos comenta Guastini Ricardo en su texto, "Sostiene que las normas que confieren derechos sociales constitucionales son engañosas porque no confieren ningún derecho verdadero. Tales formulaciones expresarían normas programáticas o teleológicas dirigidas al legislador, mediante las cuales se les 
ordenaría que persiga ciertos objetivos económico-sociales. Estas normas, no podrían garantizarse jurisdiccionalmente porque no es posible obligar al legislador a dictar leyes. La falta de promulgación de medidas legislativas tendentes a obtener tales objetivos no serían susceptible de ningún tipo de sanción." (guastini, 1999, págs. 223-224)

Podemos ver que la opinión de los dos autores citados sostiene tesis parecidas en relación a los derechos sociales, y en mi opinión personal yo me adhiero a esa misma tesis, concluyendo que el derecho a la salud no puede estar establecido en nuestra Constitución, como simplemente un derecho social, muy a pesar de haber sido elevado en el año 2015, a través de una ley estatutaria promulgada por el presidente de la república Colombiana, los intermediarios no han despertado a la realidad que la salud es vital para el ser humano, y no se está dando cumplimiento a esta norma.

\subsection{Normas Constitucionales y Legales del Derecho a la Salud}

En primera instancia are alusión a la Sentencia T-402 de 1992, donde la Corte Constitucional expide esta sentencia con el objetivo, de darle una opción a los afiliados para poder exigir su derecho a la salud, a través de la conexidad con el derecho fundamental a la vida, para poder interponer la acción de tutela de esta forma, invocando el derecho a la vida, cuando existiere un inminente riesgo de la vida de una persona.

En segundo lugar les citare la Sentencia T-760 de 2008, en esta sentencia la corte se pronuncia en el reconocimiento de la salud como derecho autónomo, autonomía que se aplica, simplemente para el beneficio de los intermediarios de la salud, y no para el objetivo principal de la corte, que es beneficiar a los usuarios del sistema de salud, para que en la prestación de los servicios se aplique la celeridad en los procedimientos de autorizaciones, para tratamientos , 
procedimientos, rehabilitación y medicamentos, y de la misma forma se presten los servicios de manera oportuna, con eficacia y calidad.

En tercer lugar le señalo la Ley 1751 de 2015, es esta la ley que eleva la salud como derecho fundamental, luego de trascurridos veintidós años de expedición de la Ley 100 de 1993, se logra tener una normatividad que ampara al derecho a la salud como derecho fundamental, muy a pesar de no estar contemplado en nuestra constitución, después de la Ley 100 de 1993, se han expedido aproximadamente, más de veinte leyes referente a la salud, las más importantes han sido; Ley 715 de 2002, Ley 1122 de 2007, Ley 1393 de 2010, Ley 1438 de 2011 y por último la Ley 1751 de 2015, que es la que estamos mencionando.

En lo que va corrido del año 2016 el Ministerio de Salud y la Protección Social, ha expedido la Resolución 0256 de 2016, resolución de gran importancia, que hace énfasis en el sistema para la calidad, y garantía de la atención a la salud, dicha resolución es de obligatorio cumplimiento, para las Empresas Promotoras de salud, las Instituciones Prestadoras de Servicios de Salud y para las Direcciones Territoriales de Salud.

Esta resolución se les aplica a las Entidades Administradoras de Planes de Beneficios (E.A.P.E.), a las Instituciones Prestadoras de Servicios de Salud (IPS), de servicios de transporte especial de pacientes, Entidades Distritales y Municipal de Salud y al Instituto Nacional de Salud.

\section{4. nueva reforma de la salud}

El objetivo principal de esta nueva reforma, se centra principalmente en garantizar el derecho a la salud, a todos los colombianos, que puedan acceder a los servicios de salud, sin la necesidad de interponer la acción de tutela, para requerir a las entidades que hacen parte del sistema, a través de un fallo judicial para poder hacer uso de procedimientos, tratamientos y medicamentos esenciales incluidos en el Plan Obligatorio de Salud POS. 
Otros de los objetivos de esta reforma son la reducción de las barreras de acceso de servicios para su calidad y continuidad dentro del sistema, de igual forma se espera, reducir la intermediación financiera, y por lo tanto con esta reducción, se lograría la transparencia, con la rendición de cuentas claras y públicas. La reforma también propone una mejor regulación y supervisión, por los órganos de dirección, vigilancia y control.

\subsection{Propuestas del Ministerio de Salud y la Protección Social}

Entre las propuestas por parte del Ministerio de Salud y la Protección Social, en la creación de nuevas entidades de salud, como áreas de gestión sanitaria, redes prestadoras de servicios de salud en tres categorías básica, especialidad y especial. Las áreas de gestión sanitarias tendrán la función de tener a su cargo la operación de la red de atención básica, y gestionara riesgos en las redes especializadas y especiales.

La financiación de la nueva reforma, se basa en recursos entregados a las redes que integran el sistema de la siguiente forma:Recibirán un giro mensual por un valor fijo, de conformidad con los servicios que presten.

- Recibirán un monto variable dependiendo de la información y el desempeño de la red.

- Recibirán un presupuesto fijo de atención básica, especializada, y especiales.

- Recibirán una unidad de pago por capitación (UPC), como ocurre en el sistema actual.

\subsection{Cuatro Formulas que Propone el Nuevo Modelo de Salud}

1) Salud Mia: Se crea como un nuevo órgano del estado para administrar los recursos del sistema de salud, remplaza al Fondo de Solidaridad y Garantía FOSYGA. 
2) Mi Plan: Este Plan de Beneficios Integral, incluiría todas las patologías, sin ninguna restricción. Además se incluiría procedimientos de alta complejidad, que no existe en todas las regiones, y se ampliaría

el potencial de medicamentos, y de esta forma no sería necesario recurrir a la interposición de la acción de tutela.

3) Gestores de Servicios de Salud: Remplazaran a las Empresas Promotoras de Salud EPS, no manejaran dinero, acompañaran a los afiliados en su paso por el sistema de salud, y responderán por la salud de sus afiliados.

4) Prestación de Servicios: Las acciones de salud pública estarán a cargo de las alcaldías y gobernaciones, y las acciones individuales estarán a cargo de los gestores de servicios de salud GES.

\section{Conclusiones}

De este artículo de investigación debemos concluir que nuestro derecho a la salud, está contemplado en la Constitución Política, entre los derechos económicos, sociales y culturales, cuando el legislador incluye un derecho básico de tan alta relevancia, como un derecho social en la constitución, está desconociendo la prevención de una garantía, para el ser humano, como lo es el derecho a la salud; estaríamos frente a la negación de la existencia del derecho mismo, y esto nos conduce, a consecuencias negativas para un sistema constitucional, nos enfrentamos a la elaboración inadecuada de una constitución, sin ningún tipo de garantía, para la subsistencia del individuo, como tal, sin salud, no hay vida, y sin vida no existimos, los derechos existen si están establecidos en la norma y las garantías existen, si de igual forma están contempladas en la normatividad, la falta de garantía debe ser considerada como un vacío total, que el estado tiene la obligación de llenar. 
En este punto sería conveniente una nueva reforma para el sistema de salud, ya que el objetivo principal de esta reforma, es garantizar el derecho fundamental a la salud, y reducir las barreras de acceso de servicios para su calidad y continuidad. Con la expedición de la Ley estatutaria 1751 de 2015, donde se eleva la salud, como derecho fundamental, y además, aprobada esta nueva reforma, la norma sería positiva, porque busca la no dilación de la prestación de los servicios de salud, como los procedimientos, tratamientos y entrega de medicamentos de una manera eficaz. De igual forma daría lugar acabar con la fragmentación y desarticulación, en lo relativo a la eficiencia financiera del sistema y no a la problemática relacionada con la atención en salud, entre los agentes de inspección, vigilancia y control, y esto nos conduciría acabar con tantas injusticias en el sistema y lograr la cobertura universal y el estancamiento en la promoción de la salud, y la prevención de enfermedades.

\section{Referencias.}

Agudelo Ibáñez, S., \& Calderón Ortega, M. (2016). Responsabilidad patrimonial del Estado colombiano por actos de reforma constitucional. Justicia, 21(29), 99-118. https://doi.org/10.17081/just.21.29.1236

Anderson, M. (2013). Testimonios de mujeres cubanas: Feminismo y afrocubanidad en tres textos de Daisy Rubiera Castillo. Cuadernos de Literatura del Caribe e Hispanoamérica, № 17.

Arango, Rodolfo "El concepto de Derechos Sociales Fundamentales" Universidad Nacional de Colombia, edición legis. Bogotá, 2006. 
Arrieta, I. (2013). 'la construcción del ethos en el discurso político: un caso de legitimación y poder', en: cuadernos de literatura del caribe e hispanoamérica, no.19. Pp.13-35. Http://investigaciones.uniatlantico.edu.co/revistas/index.php/cuadernos_literatura/article/view/124 $8 / 874$

Balcarce, F. I. (2014). DERECHO PENAL Y NEUROCIENCIA: APROXIMACIONES. Revista LEGEM, 2(1), 81-93. Retrieved from http://investigaciones.uniatlantico.edu.co/revistas/index.php/legin/article/ view/1172

Bayon, j. (1995). Derechos, democracia y Madrid: discusiones.

Bernal Pulido, Carlos "El Prinapio de Proporcionalidad y los Derechos Fundamentales" Centro de Estudios Políticos y Constitucionales, tercera edición. Madrid. 2007 y escritos sobre la aplicación de los "Derechos Fundamentales", cuarta edición, Bogotá. Universidad Externado de Colombia, 2007

Berrocal Durán, J. (2016). Igualdad material de las partes en el proceso laboral: audiencias, conciliación y primera de trámite. Justicia, 21(30), 122-131. https://doi.org/10.17081/just.21.30.1354

Caicedo Pérez, R. (2015). El derecho a huelga en Colombia: un sofisma. Legem, vol 2. Num 2., 87-100.

Carrascal, M., Chávez, C., \& Sesin, F. (2014). Correlación diagnóstica de la citología cervical versus colposcopia en lesiones premalignas de cáncer cervicouterino. IPS Universitaria Barranquilla 2013. Biociencias, 9(1), 37- 


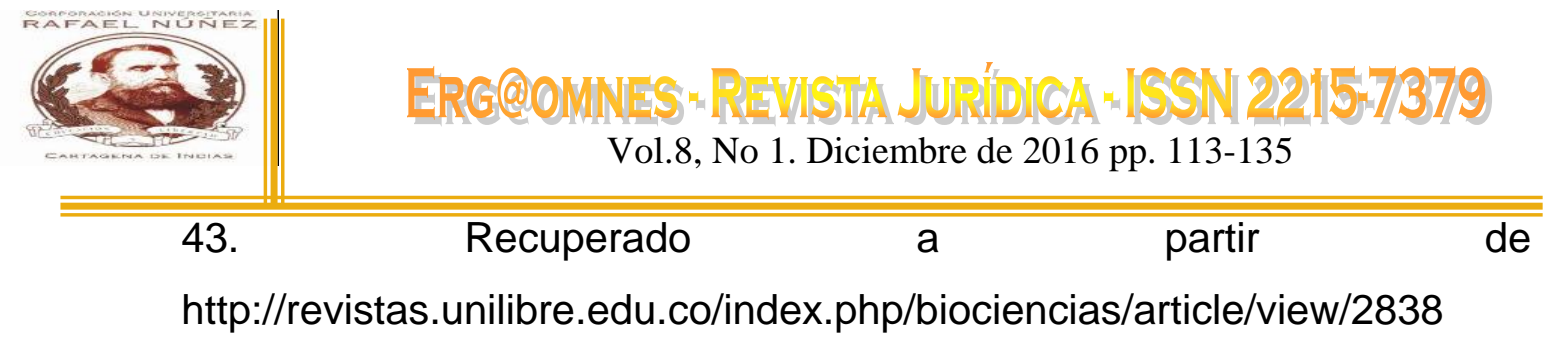

Carbonell, E. (2015). Apuntes de derecho concursal latinoamericano Colombia, Perú, México y Brasil. Revista LEGEM, 2(2), 11-35.

Díez, Adriano (2014). El estudio de la migración internacional del retorno en Colombia. Una revisión bibliográfica sobre el estado actual. Revista Amauta. $\quad$ № $24: \quad 23-\quad 39 . \quad$ Recuperado de:http://investigaciones.uniatlantico.edu.co/revistas/index.php/Amauta/a rticle/view/1065

Escudero Sabogal, I., Jam Arrieta, M., Rodríguez Arrieta, L., Rodríguez Arrieta, L., \& Rodríguez Flórez, M. (2014). Casos de lumbalgia en trabajadores de facturación central- Clínica San Juan de Dios Cartagena-2011. Biociencias, 9(2), 77-85. Recuperado a partir de http://revistas.unilibre.edu.co/index.php/biociencias/article/view/2862

Fandiño Barrios, Y. (2014). La otredad y la discriminación de géneros. Advocatus, 23, 49-57.

Ferrajoli, Luigui "Los fundamentos de los Derechos Fundamentales" Madrid. Trotta. 2001. Derechos y Garantías. Ley del más débil. Madrid: Trotta 2001.

Galettini, A. (2013). Más allá de la paradoja espacial: Otra manera de pensar la diáspora. Análisis de The Fat Black Woman's Poems, de Grace Nichols. Cuadernos de Literatura del Caribe e Hispanoamérica, $\mathrm{N}^{\circ}$. 17. 
García Cuan, A., Rosado Porto, D., \& Salas Lalinde, M. (2012). Panorama actual de los contaminantes orgánicos persistentes. Biociencias, 7(1), 81-88. Recuperado a partir de http://revistas.unilibre.edu.co/index.php/biociencias/article/view/2796

Guastini, R. (1999). Estudios de metateoria del derecho: gedisa.

Hernández, Luis,(2015) "El subjetivema y la construcción de imaginarios socioculturales". Cuadernos de Literatura del Caribe e Hispanoamérica N²1: 179-197 Enero- Junio de 2015. Universidad del Atlántico y Universidad de Cartagena, Colombia.

Morales, I. (2015). Derechos sociales constitucionales y democracia. Madrid: marcial pons.

Mosquera Rentería, J. (2015). Derechos de las minorías sexuales: retos contemporáneos de la resocialización. Justicia, 20(28), 121-138. https://doi.org/10.17081/just.20.28.1044

Morales, G., Bolaños Contreras, C., \& Larrazábal Ruiz, T. (2011). Enterobacterías aisladas en un centro hospitalario de la ciudad de Valledupar y frecuencia de betalactamasas de espectro extendido y betalactamasas inducibles. Biociencias, 6(2), 33-40. Recuperado a partir de http://revistas.unilibre.edu.co/index.php/biociencias/article/view/2778

Muñoz, F.; Bravo, M. y Blanco-Álvarez, H. (2015). Estudio sobre los factores que influyen en la pérdida de interés hacia las matemáticas. Revista Amauta. No. 26. Julio-diciembre 2015. Universidad del Atlántico. Barranquilla. 149-166. pp. Recuperado de 
http://investigaciones.uniatlantico.edu.co/revistas/index.php/Amauta/artic le/view /1328/0

Orfale, R. N. (2014). Descentralización, participación ciudadana y gobierno local en Colombia. Advocatus, 22(22), 25-40.

Osorio Gutiérrez, M. (2015). Establecimiento de comercio en relación con los hosting web. Advocatus, (24), 67-77. https://doi.org/10.18041/01240102/advocatus.24.984

Osuna Patiño, Néstor Iván "Apuntes sobre el concepto de Derechos Fundamentales. Teorías de Derecho Público. Universidad Externado de Colombia. Instituto de Estudios Constitucionales. Carlos Restrepo Piedrahita No. 37. Bogotá 1997

Pórtela, J. (2015). Construcción del consenso moral del consenso y ley natural. Justicia, 20(28), 32-55. https://doi.org/10.17081/just.20.28.1033

Reales Vega, R., de Castro Marriaga, D., \& Arcón, D. (2014). Percepción del agua como derecho fundamental: los efectos producidos por la prestación del servicio de agua potable en los habitantes del municipio de santa lucían, atlántico. Justicia, 19(26), 69-80. https://doi.org/10.17081/just.19.26.825

Rinvera García, Christian Geovany y Mondéjar Rodríguez, Juan. (2014): "La lectura crítica como habilidad investigativa en estudiantes universitarios". En: Revista Amauta. No.23, p.163-176. 
Rivera Casas, A., \& Rueda Barrios, A. (2016). Diagnóstico y prevención del parto pretermino. Una actualización de la literatura. Biociencias, 11(2), 55-66. https://doi.org/10.18041/2390-0512/bioc.2.2564

Rober, Alexi "Teoría sobre el Derecho Fundamental", segunda edición en Castellano.

Rodríguez, r. (2015). Estudios sobre seguridad social. Barranquilla: universidad del norte.

Sentencia T-180 de 1993 M.P. Hernando Herrera Vergara. Humberto Antonio Sierra Porto

Sentencia T-227 de 2003 M.P. Eduardo Montealegre.

Sentencia T-402 de 1992 Corte Constitucional.

Sentencia T-484 DE 1992 Fabio Morón Díaz.

Sentencia T-505 de 1992 M.P. Eduardo Cifuentes Muñoz. T-613 de 1992 M.P. Alejandro Martínez Caballero.

Sentencia T-536 de 1992 M.P. Simurt Rodríguez Rodríguez.

Sentencia T-561 de 1992 Corte Constitucional.

Sentencia T-760 de 2008 Corte Constitucional

Sentencia T-760 de 2008 M.P. Manuel Cepeda Espinosa. 
Sentencia T-770 y T 816 de 2008 M.P. Clara Inés Vargas Hernández.

Sentencia T-881 de 2002 M.P. Eduardo Montealegre Lyhett.

Soto, D. P. (2015). Francis Bacon y la Calumnia de America. Amauta, 1 - 16.

Vega Arrieta, H. (2015). Aspectos dogmáticos y políticos criminales de la estructura general del delito en el sistema penal colombiano. Justicia, 20(27), 42-72. https://doi.org/10.17081/just.20.27.806

Vila Casado, Iván (2007) "Fundamentos del Derecho Constitucional Contemporáneo" primera edición legis. Bogotá, 